\title{
Exact Solution of a Strongly Coupled Gauge Theory in $0+1$ Dimensions
}

\author{
Chethan Krishnan ${ }^{*}$ and K. V. Pavan Kumar ${ }^{\dagger}$ \\ Center for High Energy Physics, Indian Institute of Science, Bangalore, India
}

(Received 15 February 2018; published 16 May 2018)

\begin{abstract}
Gauged tensor models are a class of strongly coupled quantum mechanical theories. We present the exact analytic solution of a specific example of such a theory: namely, the smallest colored tensor model due to Gurau and Witten that exhibits nonlinearities. We find explicit analytic expressions for the eigenvalues and eigenstates, and the former agree precisely with previous numerical results on (a subset of) eigenvalues of the ungauged theory. The physics of the spectrum, despite the smallness of $N$, exhibits rudimentary signatures of chaos. This Letter is a summary of our main results: the technical details will appear in companion paper [C. Krishnan and K. V. Pavan Kumar, Complete solution of a gauged tensor model, arXiv:1804.10103].
\end{abstract}

DOI: 10.1103/PhysRevLett.120.201603

Introduction.-A lot of our intuition about physics is built around the harmonic oscillator because, by and large, the only equations we are able to solve are linear, and the only integrations we are able to do are Gaussian. However, it has become increasingly clear in the last half century that many of the most vexing questions in fundamental physics are likely to be solved only if we have a solid grasp of nonperturbative physics. They include things ranging from the strong coupling behavior of QCD to the black hole information paradox, just to name two. These problems are likely to take more than just technical breakthroughs to fully unravel, but part of the difficulty is certainly that we have very few examples of strongly coupled theories that are exactly solvable. As a result, we have very few models to play around with.

The purpose of this Letter is to present such an example solution to a gauged nonlinear theory of strongly interacting fermions in $0+1$ dimensions, called a "colored tensor model." We will solve this theory for a specific small value of $N$, exactly and analytically. These tensor models were first introduced in [1] because they have a large- $N$ perturbation theory that is of the same melonic type $[2,3]$ as the celebrated Sachdev-Ye-Kitaev (SYK) model [4,5]. But unlike the SYK model, there is no disorder average here, and the system is just an ordinary gauged quantum mechanics. Since large- $N$ theories are believed to be of interest for string theory and holography, our solution might shed some light on gravity at a finite value of Planck's constant [6].

Published by the American Physical Society under the terms of the Creative Commons Attribution 4.0 International license. Further distribution of this work must maintain attribution to the author(s) and the published article's title, journal citation, and DOI. Funded by SCOAP .
At a more prosaic level, what we will solve is the $O(n)^{6}$ gauged Gurau-Witten model with quartic self-interactions presented in [1] for the specific value of $n=2$ [9]. Note that we are working with a quantum mechanical theory in $0+1$ dimensions, so the gauging affects the system only via the fact that we are restricting our attention to the singlet states from the ungauged spectrum. In particular, unlike in higher dimensions, the gauge field itself does not have any dynamics and the nonlinearity comes purely from the self-interactions. This means that it is not unreasonable to hope that we can get some insight into the large- $N$ physics of this theory, even though we are looking at the Abelian $n=2$ case [10]. Indeed, we will find that this is true: the theory has a spectral form factor that shows the beginnings of the dipramp-plateau structure expected in SYK and related models at larger $N[11,12]$. This structure is believed to be related to random matrix and quantum chaos behavior [11-14].

We are able to find analytic expressions for all eigenvalues and eigenstates. We will present the details of the latter in a later paper [15], which contains substantially more detail and pages than the present Letter. Remarkably, our analytically obtained eigenvalues match precisely with numerical eigenvalues found in previous work [13] in the ungauged model up to six decimal places [16]. In particular, the ground state energy is $-2 \sqrt{14}$ in $J=1$ units. The Hilbert space we get is 140 dimensional, and the number of distinct eigenvalues is 11 . To contrast, the Hilbert space of the gauged uncolored tensor model with $n=2$ is that of a two-state system: this system was considerably simpler to obtain [14,17], and it shows no hints of chaos [12].

Our main strategy will be to take simultaneous advantage of a few different facts. One is to note that the Hilbert space of the ungauged theory is an appropriate spinor representation generalizing an observation in [18] for the uncolored model. This alone is not sufficient to make the problem 
tractable, however. But when one restricts attention to gauge singlet states, one finds a remarkable simplification: that only the mid-Clifford level remains. Even this is a hard problem still, but with a judicious application of discrete symmetries and brute force, we find that in the $n=2$ case it becomes surmountable. In what follows, we will discuss $S O(n)^{6}$ as our gauge group for concreteness and will be cavalier about distinguishing between $O(n)$ and $S O(n)$. We have also considered the gauged $O(n)^{6}$ case, which removes some of the singlets and eigenstates we find here. These will be discussed in [15].

The model.-The quartic version of the Gurau-Witten (GW) model is constructed using fermionic tensors of the form $\psi_{A}^{i j k}$ where $A$ denotes the color and takes values $\{0,1,2,3\}$. For every pair of colors $(A, B)$, we assign a group $O(n)$; i.e., the overall symmetry group of the theory is

$$
\begin{aligned}
G \sim & O(n)_{01} \times O(n)_{02} \times O(n)_{03} \times O(n)_{12} \\
& \times O(n)_{13} \times O(n)_{23} .
\end{aligned}
$$

Under any of the above orthogonal groups, exactly two of the fermionic tensors transform in the fundamental representation.

The Lagrangian of the GW model is a scalar with respect to the symmetry group $G$ and is given by

$$
\mathcal{L}=\frac{i}{2} \psi_{A}^{i j k} \partial_{t} \psi_{A}^{i j k}+\frac{J}{n^{3 / 2}} \sum \psi_{0}^{i j k} \psi_{1}^{i l m} \psi_{2}^{n j m} \psi_{3}^{n l k} .
$$

Here $J$ is a dimensionful coupling and we set $J=1$ from now on [19]. Further, quantizing the theory leads to the following anticommutation relations

$$
\left\{\psi_{A}^{i j k}, \psi_{B}^{p q r}\right\}=\delta_{A B} \delta^{i p} \delta^{j q} \delta^{k r}
$$

For later purposes, we compute the Noether charges corresponding to the symmetry group $G$. They are given by

$$
\begin{aligned}
& Q_{01}^{i_{1} i_{2}}=i\left(\psi_{0}^{i_{1} j k} \psi_{0}^{i_{2} j k}+\psi_{1}^{i_{1} j k} \psi_{1}^{i_{2} j k}\right), \\
& Q_{23}^{i_{1} i_{2}}=i\left(\psi_{2}^{i_{1} j k} \psi_{2}^{i_{2} j k}+\psi_{3}^{i_{1} j k} \psi_{3}^{i_{2} j k}\right), \\
& Q_{02}^{j_{1} j_{2}}=i\left(\psi_{0}^{i j_{1} k} \psi_{0}^{i j_{2} k}+\psi_{2}^{i j_{1} k} \psi_{2}^{i j_{2} k}\right), \\
& Q_{13}^{j_{1} j_{2}}=i\left(\psi_{1}^{i j_{1} k} \psi_{1}^{i j_{2} k}+\psi_{3}^{i j_{1} k} \psi_{3}^{i j_{2} k}\right), \\
& Q_{03}^{k_{1} k_{2}}=i\left(\psi_{0}^{i j k_{1}} \psi_{0}^{i j k_{2}}+\psi_{3}^{i j k_{1}} \psi_{3}^{i j k_{2}}\right), \\
& Q_{12}^{k_{1} k_{2}}=i\left(\psi_{1}^{i j k_{1}} \psi_{1}^{i j k_{2}}+\psi_{2}^{i j k_{1}} \psi_{2}^{i j k_{2}}\right),
\end{aligned}
$$

where $Q_{A B}$ denotes the Noether charge corresponding to the group $O(n)_{A B}$. Also, we note that the twin upper indices on any of these charges should not be equal.
Apart from being an invariant under the symmetry group $G$, the interaction term of the Lagrangian (i.e., the Hamiltonian) also has some discrete symmetries. These symmetries are related to the permutation of colors and are not a part of the symmetry group $G$ that we will gauge in the later parts of the Letter. These discrete symmetries are helpful in reducing the number of computations we need to do considerably. We refer the reader to [15] for a detailed discussion on these discrete symmetries.

The ungauged Hilbert space.-Our goal is to find the singlet spectrum of the simplest $(n=2)$ quartic $\mathrm{GW}$ model. An analogous discussion corresponding to the $n=2$ uncolored model has appeared in [14], which utilizes the technology developed in [18]. It was found that the ground state and highest energy state constitute the singlet spectrum of the $n=2$ uncolored model. Even though the discussion was for $n=2$, the strategy presented there would work for any general even $n$. In this Letter, we generalize that strategy to the colored GW model and apply it to the case of $n=2$. As we will see, unlike the uncolored version, the singlet spectrum of the $n=2 \mathrm{GW}$ model is highly nontrivial and also shows signs of chaos.

Let us now outline our strategy. We start by defining the basis for the Hilbert space that we work with. Slightly generalizing [18], we exploit the Clifford structure and define the colored creation and annihilation operators as follows:

$$
\psi_{A}^{i j k^{ \pm}}=\frac{1}{\sqrt{2}}\left(\psi_{A}^{i j k} \pm i \psi_{A}^{i j(k+1)}\right),
$$

where the indices $k^{ \pm} \in\{1, n / 2\}$ and are given by $k=2 k^{ \pm}-1$. The basis is constructed starting with the lowest weight state (or the Clifford vacuum) |\rangle that is annihilated by all the annihilation operators, i.e., $\psi_{A}^{i j k^{-}}|\rangle=0$. Now, we can act with the creation operators on the Clifford vacuum to generate the entire Hilbert space. As the number of creation operators is $2 n^{3}$, the dimensionality of the Hilbert space is $2^{2 n^{3}}$. Note that the Clifford vacuum and states are entirely distinct from the eigenstates of the Hamiltonian. In fact, we will find that the entire singlet spectrum lies in the midClifford level, so the tendency to conflate the two bases should be strongly resisted.

For later purposes, we define the level operators corresponding to each of the colors as follows:

$$
L_{A}=\sum \psi_{A}^{i j k^{+}} \psi_{A}^{i j k^{-}} .
$$

Note that the color index $A$ is not summed over on the rhs. It is straightforward to verify that the Hamiltonian commutes with the overall level operator $\sum_{A} L_{A}$, but not with level operators of individual colors.

Now that we have a basis, the next step is to identify the singlet states. The singlet states, by definition, are the states that have a zero charge under the symmetry group $G$. On an operational level, this requirement translates to the 
statement that the Noether charges (4)-(9) annihilate the singlet states. That is, we need to find a generic linear combination of our basis states that are annihilated by the Noether charges. Starting with the condition that the charges $Q_{03}$ and $Q_{12}$ annihilate the singlet states, we can show the following:

$$
\left.\left.\left.\left(L_{0}+L_{3}\right) \mid \text { singlet }\right\rangle=\left(L_{1}+L_{2}\right) \mid \text { singlet }\right\rangle=\frac{n^{3}}{2} \mid \text { singlet }\right\rangle .
$$

This implies that all the singlet states are at the mid-Clifford level, i.e., at level $n^{3}$ with $n^{3} / 2$ fermions belonging to the colors 0 and 3 , and the other $n^{3} / 2$ of them belonging to the colors 1 and 2 .

Once we find the singlet states, the next step will be to determine which combinations of singlet states are eigenstates of the Hamiltonian. Since the Noether charges commute with the Hamiltonian, we are guaranteed that acting with Hamiltonian on any singlet state gives a combination of singlet states.

The gauged Hilbert space.-From here on, we deal with the specific case of $n=2$. There are four creation operators of each color and hence the dimensionality of the ungauged Hilbert space is $2^{16}$. The midlevel condition implies that all the singlets are at the eighth level, with four of the creation operators belonging to the colors 0 and 3 , whereas the other four belong to the colors 1 and 2. These states can be divided into various groups based on the bipartitions of 4 . We denote these partitions by $p_{1}, \ldots, p_{5}$ and, more specifically, we have

$$
p_{1}: 4+0 ; \quad p_{2}: 3+1 ; \ldots ; \quad p_{5}: 0+4 .
$$

Every state that satisfies the midlevel condition belongs to exactly one of the groups denoted by an ordered pair ( $p_{i}$, $p_{j}$ ). The first partition $p_{i}$ corresponds to the partition of the colors 0 and 3 , and the second partition $p_{j}$ corresponds to the partition of colors 1 and 2. Note that there are 4900 states in the (sub-)Hilbert space satisfying the midlevel condition and these states are divided into 25 different groups labeled by $\left(p_{i}, p_{j}\right)$.

Before proceeding further, let us write down the Noether charges in terms of $\psi^{ \pm}$'s as follows:

$Q_{01}^{12}=i\left(\psi_{0}^{1 j+} \psi_{0}^{2 j-}-\psi_{0}^{2 j+} \psi_{0}^{1 j-}+\psi_{1}^{1 j+} \psi_{1}^{2 j-}-\psi_{1}^{2 j+} \psi_{1}^{1 j-}\right)$,

$Q_{23}^{12}=i\left(\psi_{2}^{1 j+} \psi_{2}^{2 j-}-\psi_{2}^{2 j+} \psi_{2}^{1 j-}+\psi_{3}^{1 j+} \psi_{3}^{2 j-}-\psi_{3}^{2 j+} \psi_{3}^{1 j-}\right)$,

$Q_{02}^{12}=i\left(\psi_{0}^{i 1+} \psi_{0}^{i 2-}-\psi_{0}^{i 2+} \psi_{0}^{i 1-}+\psi_{2}^{i 1+} \psi_{2}^{i 2-}-\psi_{1}^{i 2+} \psi_{1}^{i 1-}\right)$,

$Q_{13}^{12}=i\left(\psi_{1}^{i 1+} \psi_{1}^{i 2-}-\psi_{1}^{i 2+} \psi_{1}^{i 1-}+\psi_{3}^{i 1+} \psi_{3}^{i 2-}-\psi_{3}^{i 2+} \psi_{3}^{i 1-}\right)$.

Note that all the charges we have listed here commute [20] with the level operators (11). The other two charges have a simple form in the $n=2$ case and are given by

$$
Q_{03}=L_{0}+L_{3} ; \quad Q_{12}=L_{1}+L_{2} .
$$

Note that the midlevel condition is the only information we obtain from these two charges in this particular case of $n=2$. Further, these two charges commute with the level operators. All the Noether charges commuting with the level operators is one of the important simplifications that happen in the case of $n=2$. This allows us to consider the singlets of each group separately.

To find the singlets, we proceed as follows. We start with a generic candidate singlet state of the form

$$
\begin{aligned}
& \sum \alpha_{i_{1} j_{1} ; i_{2} j_{2} ; \ldots ; i_{8} j_{8}}^{0 / 3,1 / 2} \psi_{0 / 3}^{i_{1} j_{1} 1^{+}} \psi_{0 / 3}^{i_{2} j_{2} 1^{+}} \psi_{0 / 3}^{i_{3} j_{3} 1^{+}} \psi_{0 / 3}^{i_{4} j_{3} 1^{+}} \\
& \quad \times \psi_{1 / 2}^{i_{5} j_{5} 1^{+}} \psi_{1 / 2}^{i_{6} j_{6} 1^{+}} \psi_{1 / 2}^{i_{7} j_{j} 1^{+}} \psi_{1 / 2}^{i_{8} j_{8} 1^{+}}|\rangle,
\end{aligned}
$$

where we need to determine $\alpha$ 's such that all the Noether charges (14) annihilate this state. The following observation is useful. The states that are annihilated by the Noether charges (14) have a zero charge under the respective orthogonal groups. The Clifford vacuum we are working with, by definition, is invariant under $S O(2)^{4} \times U(1)^{2}$ and the charges (14) correspond to these four orthogonal groups. As a result, the creation operators acting on the Clifford vacuum in a singlet state should necessarily be invariants [21] of $O(2)_{01} \times O(2)_{23} \times O(2)_{02} \times O(2)_{13}$. The only invariant tensors of the orthogonal group are the Kronecker delta and the Levi-Civita tensor. This implies that the $\alpha$ 's should be made up of $\delta$ 's and $\epsilon$ 's. This observation combined with the midlevel condition suffices to list down all the singlets of the $n=2 \mathrm{GW}$ model. Note, however, that this description is redundant.

Another way to find singlet states is by doing a brute force calculation. We start with (16) and then find $\alpha$ 's by demanding that all the Noether charges annihilate this state. We expect that both the above approaches lead to the same set of singlets, but in the following, we are using the results of this second approach. More details of this computation and a comparison with the first approach will be presented in a later paper [15].

The end result is that there are a total of 140 singlet states and they are given explicitly in one of the appendices of [15]. Out of the total 25 groups, singlets are present in only 13 of them, as summarized in Table I. We finally note that the discrete symmetries of the Hamiltonian are helpful in identifying the singlets in both the methods and also in

\begin{tabular}{|c|c|c|c|c|c|c|}
\hline Group & $\left(p_{1,5}, p_{1,5}\right.$ & $p_{1,5}, p_{3}$ & $\left(p_{3}, p_{1,5}\right.$ & $p_{2,4}, p_{2,4}$ & $p_{3}, p$ & Total \\
\hline Singlets & $4 \times 1$ & $2 \times 4$ & $2 \times 4$ & $4 \times 16$ & 56 & 140 \\
\hline
\end{tabular}
reducing the number of computations in the latter case. For more details, we refer the reader to [15].

TABLE I. Singlets present in various groups. All the groups that are not listed here have zero singlets. 
Singlet eigenstates. - In the last section, we have found all the singlets in the $n=2 \mathrm{GW}$ model. The next step is to identify the eigenstates and eigenvalues of the Hamiltonian from the singlets. We start by reorganizing the Hamiltonian of the $n=2 \mathrm{GW}$ model

$$
\begin{aligned}
H= & \psi_{0}^{i j+} \psi_{1}^{i l+} \psi_{2}^{n j-} \psi_{3}^{n l-}+\psi_{0}^{i j+} \psi_{1}^{i l-} \psi_{2}^{n j+} \psi_{3}^{n l-} \\
& +\psi_{0}^{i j-} \psi_{1}^{i l+} \psi_{2}^{n j-} \psi_{3}^{n l+}+\psi_{0}^{i j-} \psi_{1}^{i l-} \psi_{2}^{n j+} \psi_{3}^{n l+} .
\end{aligned}
$$

Although it is conceptually straightforward to act with this Hamiltonian and identify the eigenstates, the computations involved are quite tedious. Note, however, that had we not been able to usefully list the singlets, the calculation would not just be cumbersome, but impossible. An additional complication (as compared to the calculation to find singlets) is that the Hamiltonian does not commute with the level operators (11) and hence its action on a singlet of some group gives rise to singlets of other groups. Again, the discrete symmetries of the Hamiltonian are helpful here to reduce the number of computations we need to do.

We find that all the 140 eigenstates are divided into 16 independent sets. An independent set is defined as follows. The Hamiltonian acting on any singlet in the independent set gives rise to combination of singlets in the same set. The details of how we use the set structure to construct eigenstates is technical and somewhat tangential to our present purposes, so it will be presented in [15].

We conclude this section by giving an overview of the singlet spectrum. The spectrum has a spectral mirror symmetry and the degeneracy increases towards the zero energy (midlevel energy). The ground state or the lowest energy state has an energy of $-2 \sqrt{14}$ in units where the coupling is unity. Note that the ground state is unique. This was a feature also shared by the ungauged model [13]. This means that the eigenvalues of the two ground states should match. Happily, we find that the lowest energy value obtained by numerically diagonalizing the $n=2 \mathrm{GW}$ model [13] is indeed $-2 \sqrt{14}$ up to six decimals. Indeed all the eigenvalues we have obtained have counterparts in the numerical diagonalization. This is a nontrivial check of our results. The eigenvalues are summarized in Table II. For more details and also for the complete set of eigenstates, we refer to [15].

Under all the known discrete symmetries [1], the ground state transforms into itself. This gives a nontrivial check for our spectrum as some of the discrete symmetries act quite nontrivially on the singlets. Among the degeneracies we find in the spectrum, we have checked that some are explained by the known [1] discrete symmetries of the

TABLE II. Eigenvalues and corresponding degeneracy of the singlet eigenstates.

\begin{tabular}{lcccccc}
\hline \hline Eigenvalue & $\pm 2 \sqrt{14}$ & $\pm 4 \sqrt{3}$ & $\pm 2 \sqrt{6}$ & \pm 4 & $\pm 2 \sqrt{2}$ & 0 \\
\hline Degeneracy & 1 & 3 & 4 & 6 & 31 & 50 \\
\hline \hline
\end{tabular}

Gurau-Witten model. In particular, we can explain the degeneracies of all the eigenvalues except 0 and $\pm 2 \sqrt{2}$ using these discrete symmetries. The fact that there are some leftover degeneracies suggests that there are some accidental symmetries at those levels, which have hitherto not been identified. It will be interesting to identify them.

Note also that finding the spectrum is the hard part. Once we do, it is rather trivial to check that the states are eigenstates with the listed eigenvalues. So we can have considerable confidence that our results are correct [23].

Chaos and hints of large N.-In this section, we do a preliminary study of the spectrum and find evidence for chaos [11-13], which is expected at large $N$. The tool we use is the spectral form factor (SFF) [11]. It is defined as follows:

$$
F(\beta, t)=\left|\frac{Z(\beta, t)}{Z(\beta, 0)}\right|^{2} ; \quad Z(\beta, t)=\operatorname{Tr}\left(e^{-(\beta+i t) H}\right) .
$$

Cotler et al. [11] have argued that the SFF computed for a fixed inverse temperature $\beta$ and plotted as a function of time for SYK should have a dip-ramp-plateau structure, and that it is a hint of random matrixlike behavior. Using numerical diagonalization, it was shown in [13] that the SFF corresponding to the $n=2$ ungauged GW model also has a similar structure. This is perhaps unsurprising because the number of eigenvalues in the ungauged model is big: 65536 . In the gauged model here, on the other hand, where the Hilbert space dimensionality is smaller (140) and the number of distinct eigenvalues is just 11, it is not obvious that we should see hints of chaos. We compute the SFF for $\beta=0.5$ and plot it after a sliding-time average with different window sizes $\Delta t$ (see [14] for technical details of how to do this) in Fig. 1. We find that a rudimentary dipramp-plateau structure exists as in the ungauged models $[12,13]$, suggesting that the gauged sector of the $n=2 \mathrm{GW}$ model could be chaotic [25]. This can be thought of as encouraging, for those with holographic intentions for this model. But note, however, that since the number of distinct eigenvalues we have is only 11, it is not meaningful to investigate the level spacing statistics.

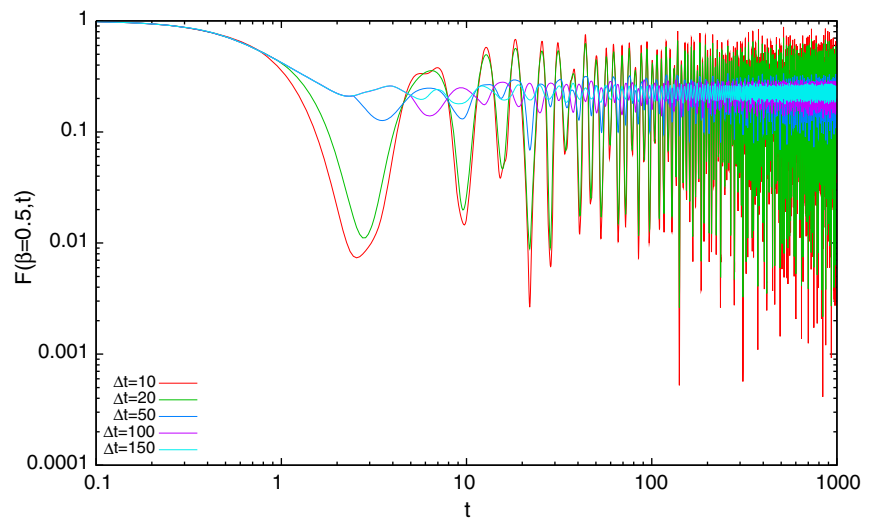

FIG. 1. SFF for the singlet spectrum of the $n=2$ Gurau-Witten model for $\beta=0.5$. 
There are a few different lines of investigations that one can undertake with a (potentially) holographic theory whose complete set of eigenvalues and eigenvectors are known: some of these will be reported elsewhere. It will also be very interesting to adapt the approach here to the simpler uncolored model [27] for arbitrary values of $N$ [22].

We thank Avinash Raju for a related collaboration and Diptiman Sen for discussions.

*chethan.krishnan@gmail.com

†umar.pavan56@gmail.com

[1] E. Witten, An SYK-like model without disorder, arXiv:1610 .09758 .

[2] R. Gurau, Invitation to random tensors, SIGMA 12, 094 (2016); R. Gurau and J. P. Ryan, Colored tensor models-a review, SIGMA 8, 020 (2012); V. Bonzom, R. Gurau, and V. Rivasseau, Random tensor models in the large $N$ limit: Uncoloring the colored tensor models, Phys. Rev. D 85, 084037 (2012); S. Carrozza and A. Tanasa, $O(N)$ random tensor models, Lett. Math. Phys. 106, 1531 (2016).

[3] V. Bonzom, L. Lionni, and A. Tanasa, Diagrammatics of a colored SYK model and of an SYK-like tensor model, leading and next-to-leading orders, J. Math. Phys. (N.Y.) 58, 052301 (2017); P. Narayan and J. Yoon, SYK-like tensor models on the lattice, J. High Energy Phys. 08 (2017) 083; S. Dartois, H. Erbin, and S. Mondal, Conformality of $1 / N$ corrections in SYK-like models, arXiv:1706.00412; R. de Mello Koch, R. Mello Koch, D. Gossman, and L. Tribelhorn, Gauge invariants, correlators and holography in bosonic and fermionic tensor models, J. High Energy Phys. 09 (2017) 011; J. Yoon, SYK models and SYK-like tensor models with global symmetry, J. High Energy Phys. 10 (2017) 183; S. Prakash and R. Sinha, A complex fermionic tensor model in $d$ dimensions, J. High Energy Phys. 02 (2018) 086.

[4] J. Polchinski and V. Rosenhaus, The spectrum in the Sachdev-Ye-Kitaev model, J. High Energy Phys. 04 (2016) 001.

[5] J. Maldacena and D. Stanford, Remarks on the Sachdev-YeKitaev model, Phys. Rev. D 94, 106002 (2016).

[6] But there are various subtleties regarding this, see, for example, $[7,8]$.

[7] J. Murugan, D. Stanford, and E. Witten, More on supersymmetric and 2d analogs of the SYK model, J. High Energy Phys. 08 (2017) 146.

[8] S. Choudhury, A. Dey, I. Halder, L. Janagal, S. Minwalla, and R. Poojary, Notes on melonic $O(N)^{q-1}$ tensor models, arXiv: 1707.09352.

[9] Note that this $n$ should not be blindly thought of as the $N$ that is relevant for the large- $N$ limit. The spinors of this theory are those of $S O(32)$, so in a SYK-like language, the $N$ here is 32. But it should be kept in mind that the scaling of the coupling $J$ in this theory is different from that in SYK, so they have to be compared with care. See [1] and various follow-ups for a discussion on the precise large- $N$ limit in these models.

[10] This assumes that we find a sufficiently large number of singlet states in the spectrum after the gauging. It turns out that the gauged Hilbert space is 140 dimensional and that there are 11 distinct eigenvalues.

[11] J. S. Cotler, G. Gur-Ari, M. Hanada, J. Polchinski, P. Saad, S. H. Shenker, D. Stanford, A. Streicher, and M. Tezuka, Black holes and random matrices, J. High Energy Phys. 05 (2017) 118.

[12] C. Krishnan and K. V. Pavan Kumar, Complete solution of a gauged tensor model, arXiv:1804.10103.

[13] C. Krishnan, S. Sanyal, and P. N. Bala Subramanian, Quantum chaos and holographic tensor models, J. High Energy Phys. 03 (2017) 056.

[14] C. Krishnan, K. V. Pavan Kumar, and D. Rosa, Contrasting SYK-like models, J. High Energy Phys. 01 (2018) 064.

[15] C. Krishnan and K. V. Pavan Kumar, Complete analytic solution of a gauged tensor model (to be published).

[16] Note that the ungauged theory has more states than the gauged theory. But the eigenvalues of the former should appear as a subset of the latter. This is indeed what we find. The eigenvalues of the ungauged model are easy to obtain by diagonalizing the Hamiltonian matrix numerically on a computer.

[17] S. Chaudhuri, V. I. Giraldo-Rivera, A. Joseph, R. Loganayagam, and J. Yoon, Abelian tensor models on the lattice, Phys. Rev. D 97, 086007 (2018).

[18] C. Krishnan and K. V. P. Kumar, Towards a finite- $N$ hologram, J. High Energy Phys. 10 (2017) 099.

[19] Setting $J=1$ is a choice of unit because $J$ is dimensionful, so we can reinstate in at any stage by dimensional analysis. The theory we solve is the UV theory, and one can reproduce IR physics by calculating correlators explicitly and looking at their late time behavior. This will lead to an effective running coupling.

[20] The charges (4)-(7) commute with the level operators for arbitrary $n$.

[21] Note that this discussion about the first four Noether charges (4)-(7) can be generalized for any arbitrary $n$, as long as we are working with the basis we have introduced here. A similar discussion for the uncolored model with a generic $n$ will be presented in [22].

[22] C. Krishnan, K. V. P. Kumar, and A. Raju (to be published).

[23] Recently, preprint [24] appeared on the arXiv. They present an indirect way to count the number of singlets in tensor models, and the number they find for the $n=2$, $D=3 \mathrm{GW}$ model is 140 , in agreement with our explicit construction.

[24] I. R. Klebanov, A. Milekhin, F. Popov, and G. Tarnopolsky, On the spectra of eigenstates in fermionic tensor quantum mechanics, arXiv:1802.10263.

[25] The qualitative similarity between the SFF of gauged and ungauged models can be viewed as supporting [26]. However, note that the claim there is that, in the holographic dual, the low curvature parts are identical for both the gauged and ungauged models. To show this conclusively one should construct the holographic dual of these tensor models, which is still an open problem.

[26] J. Maldacena and A. Milekhin, To gauge or not to gauge?, arXiv:1802.00428.

[27] I. R. Klebanov and G. Tarnopolsky, Uncolored random tensors, melon diagrams, and the Sachdev-Ye-Kitaev models, Phys. Rev. D 95, 046004 (2017). 